\title{
Visualization and Analysis of Lighting Design Alternatives in Simulation Software
}

\author{
Diana Fernandez-Prieto ${ }^{1, a^{*}}$, Hans Hagen ${ }^{1, b}$ \\ ${ }^{1}$ Computer Graphics and $\mathrm{HCl}$ group, University of Kaiserslautern, Germany \\ afprieto@cs.uni-kl.de, 'bagen@cs.uni-kl.de
} Keywords: Lighting simulation, lighting design process, visualization techniques, analysis
techniques.

\begin{abstract}
For decades, multiple lighting simulation software packages and plugins for commercial software have been developed in an effort to ease the usage and integration of simulation into the lighting design process. In this effort, one of the main challenges is to provide lighting designers with an easy and comprehensive access to simulation results. Visualization is used as a means to achieve this goal. In this paper, we explore two of the most used free lighting simulation packages towards the identification of visualization techniques that facilitate the access to the simulation results as well as the identification of opportunities for the enhancement of simulation-assisted lighting design processes. A test case of a metal workshop illustrates the output produced by both software packages. Based on this exploration, we identify an open gap regarding three main aspects: interactive exploration of simulation results, visualization of compliance with lighting standards, and visual comparison of lighting solutions. We provide a discussion on how approaches from other domains can be applied to close this gap.
\end{abstract}

\section{Introduction}

With the increasing popularity of computer-aided design tools as well as the need to satisfy energy efficiency, visual comfort, and aesthetics requirements, lighting simulation has become an essential tool in building design. From the aesthetics point of view, lighting simulation is used to illustrate how a certain design favors the utilization of natural and electric light, and from a functional point of view, it is used to predict and evaluate performance-related aspects. Both, aesthetics and functional aspects have been widely studied from several domains [1-3] in order to contribute to the formulation of guidelines for lighting designers. These guidelines are usually compiled in the form of standards such as [4]-[6] that describe ideal lighting conditions to carry out different types of tasks for different applications (e.g., office-related activities, sports and recreation activities, industrial activities, etc.).

For over three decades, a great effort has been dedicated to the development of computational models that describe as accurate as possible the complex behavior of light and its interaction with the environment. Although these models are ever more accessible to a larger audience, their actual integration into the building design process is still challenging. Two of the main obstacles for this integration is the ease-of-use of these models and the ability to interpret simulation results effectively [7] [8]. To tackle the ease-of use obstacle, several software packages in the architecture industry have incorporated these models within CAD tools like Ecotect, ElumTools for Autodesk Revit, Diva for Rhino, and 3DS Max among others. Likewise, specific lighting simulation software packages (e.g., Agi32, ADELINE, DIALux, Relux, Lightscape, etc.) have been developed with the purpose of providing a user-friendly interface for the utilization of these models. Regarding the interpretation and comparison of simulation results, visualization and analytics techniques are used to facilitate the understanding of lighting behavior in buildings. However, as new techniques become available (e.g., ensemble visualization, image processing, etc.), this is a matter open to further improvement.

In this paper we explore the output provided by two of the most used free lighting simulation software packages with a view to identifying the visualization and analysis techniques that support 
the lighting design process. First, we introduce the related work. Then, we outline the lighting simulation process from a decision-making perspective and highlight some of the most important metrics that are considered when planning a lighting solution. Later on, we illustrate through a test case, the output and analysis techniques provided by both tools focusing on three main criteria: 1) visualization and analysis alternatives for the exploration of simulation results, 2) support for the validation of international standards, and 3) comparison of lighting solutions. Finally, we discuss on how visualization and analytics could be improved, and we provide a brief overview on the techniques used in other fields that could be applied in this application domain to enhance the insights gained from lighting simulation.

\section{Related Work}

Several studies have been dedicated to the comparison of different lighting simulation software. While some of them focus on the performance and accuracy of the calculation methods, and others discuss usability aspects [8-10], only a few discuss the visualization and analysis of the simulation results. In this section, we only make reference to the studies that include to some extent the simulation output interpretation.

For instance, Roy [11] does not only discuss the computational methods used in four simulation packages (ADELINE, Lightscape, Microstation, and RadioRay) but also comments on the alternatives offered by these tools to present the simulation results. Also analyzed by Ashmore and Richens [12], these alternatives respond to the need of designers to interpret photometric data in a graphic way. Some of the visualization techniques listed by these two studies include: isocontours, false-color surfaces, and photorealistic rendering. Ochoa et al. [13] provide a detailed review on the state of the art of lighting simulation in building design. They classify the output provided by simulation tools in two categories: quantitative and qualitative output. The quantitative output consists basically of text-only files that contain photometric data (e.g., illuminance, luminance, glare, etc.). The qualitative output is expressed as different types of image output that depict a mapping from the quantitative output to a graphic representation. Iversen et al. [14] compare nine lighting simulation packages in the context of daylighting simulation. Despite the analysis focuses on the accuracy of daylight computation for each tool, it also presents the alternatives for the graphical representation of daylight factor levels. Once again, iso-contours and false-color maps along with tabular data are the preferred techniques by most of the tools for making the simulation results available to lighting designers. Similar studies comparing other tools can be found in [15][16].

Although not in the lighting simulation domain but in the building performance simulation domain, Prazeres and Clarke [17] address the communication of simulation output to the users. Considering the fact that in most cases simulation output is heterogeneous with respect to the data type, a reasonable approach is to offer specialized visualizations according to the type of data that needs to be displayed. The authors of this paper present a survey of visualization techniques that can be used in the context of the visualization of building simulation results. Among these, interactive 3D visualizations and virtual reality environments are highlighted as they allow the exploration of the results from different points of view. Srivastav et al. [18] argue that even when visualization techniques have been successfully applied to the design and presentation of building designs, in the field of building simulation, there is still a gap in the application of these techniques at the service of more complex analytical tasks (e.g., analysis of multivariate data). Therefore, approaches from domains like scientific visualization should be considered and properly adapted in the context of building or lighting simulation.

In this direction, Haeb et al. [19] perform a simple study comparing the visualization capabilities of two simulation tools (IESVE and DesignBuilder, also capable of computing simple lighting simulations). Here, the authors identify visualization techniques used in other application domains that could be used for the visualization and analysis of indoor environment simulations (mostly thermal conditions). This is an analogous study to the intension of our study since it 
highlights additional visualization and analysis techniques that can potentially enhance the interpretation of simulation results.

\section{Tasks in Simulation-Assisted Lighting Design}

Simulation-assisted lighting design should be seen as an iterative process where a lighting solution is iteratively modified until certain quality criteria are met. Based on the documentation in [20] [21] we derived the steps comprised in a typical simulation-assisted lighting design process:

1. Identify lighting requirements. This step includes the analysis of the physical space to be illuminated and the extraction of lighting requirements based on the type of task to be performed in each area of the physical space. Lighting standards provide recommendations and minimum requirements with respect to illuminance levels, glare levels, light uniformity, and light color rendering index for different types of visual task areas.

2. Select lighting equipment. The selection of lamps, luminaires and control equipment, is done based on the requirements identified in the previous step and technical specifications provided by the manufacturers (commonly provided as photometric data files).

3. Model the physical space. 3D modeling tools are used to create a virtual model of the space to be illuminated. This model describes the spatial distribution of objects (e.g., furniture, openings, machines, etc.), the optical properties all surfaces within the model (materials definition), the subdivision of the space according to visual task types, and the proposed arrangement of luminaires.

4. Setup and run simulation. This step requires the definition of input data and calculation objects. The input data consists of the 3D model and photometric files of the selected luminaires. The calculation objects consist of a set of geometric elements that serve as sensors for the computation and storage of simulation results. Relevant aspects for the configuration of these objects are their position and resolution (when a calculation plane is defined).

5. Analyze the results. The impact of a particular design is analyzed in terms of performance, comfort, and safety. This analysis can lead to modifications in the original design and therefore a new design and simulation cycle must be performed.

Typical simulation-assisted lighting design processes require conducting multiple simulation cycles and thus, the task of comparing alternatives is crucial to find an optimal solution. The goal of this task is to determine, among a collection of simulations, which one fulfills more appropriately a set of requirements. To this end, we use as a reference framework the European standard EN 12464-1 "Lighting of work places - Indoor work places" [4]. According to this standard, any lighting design must satisfy three basic human needs: 1) visual comfort, 2) visual performance, and 3) safety. Qualitative and quantitative metrics are used to evaluate whether these needs are successfully fulfilled. In this study we focus on the following quantitative metrics:

- Luminance $(L)$. It refers to the amount of light reflected from a surface. It can also be interpreted as the perceived brightness of a surface based on its reflectance and the illuminance on it. Luminance has a direct impact on the visual comfort and visual performance as it can affect task visibility.

$$
L_{V}=\frac{d^{2} \Phi_{V}}{d \Omega * d \Sigma * \cos \theta}
$$

where:

$d^{2} \Phi_{V} \quad$ is the luminous flux leaving the area $d \Sigma$ in any direction contained inside the solid angle $d \Omega$.

$d \Omega \quad$ is an infinitesimal solid angle containing the specified direction.

$d \Sigma \quad$ is an infinitesimal area $\left(\mathrm{m}^{2}\right)$ of the source containing the specified point.

$\theta \quad$ is the angle between the normal of the surface and the specified direction. 
- Illuminance $(E)$ and Maintained Illuminance $\left(\bar{E}_{m}\right)$. Illuminance refers to the amount of light that falls onto a surface. A related term is the maintained illuminance which refers to the value below which the illuminance level must not fall. The standard EN 12464-1 provides reference values for this metric in accordance to the type of visual task. This metric is essential to visual performance.

$$
\mathrm{E}=\frac{\Phi_{V}}{A}
$$

where:

$\Phi_{V} \quad$ is the luminous flux measured in lumens.

$A \quad$ is the area hit by luminous flux measured in $\left(\mathrm{m}^{2}\right)$.

- Unified Glare Rating (UGR). It is a metric to estimate the glare sensation perceived by humans. It takes into account the total amount of luminaires as well as the optical properties of the materials in the visual task area. This metric impacts the visual performance and security as glare effects can cause errors during the performance of the visual task, fatigue, and accidents. A UGR below 10 is imperceptible for the human eye while a UGR over 30 indicates a very high glare which becomes uncomfortable for the human eye.

$$
\mathrm{UGR}=8 \log \left(\frac{0.25}{L_{b}} \Sigma \frac{L^{2} \Omega}{P^{2}}\right)
$$

where:

$L_{b} \quad$ is the background luminance $\left(\mathrm{cd} / \mathrm{m}^{2}\right)$.

$L \quad$ is the luminance of the luminous parts of each luminaire in the direction of the observer's eye $\left(\mathrm{cd} / \mathrm{m}^{2}\right)$.

$\Omega \quad$ is the solid angle of the luminous parts of each luminaire at the observer's eye.

$P \quad$ is the Guth position index for each luminaire (displacement from the line of sight).

- Uniformity Index $\left(U_{o}\right)$. To favor a suitable adaptation luminance, a task area should be illuminated homogeneously. Therefore the illuminance in the task area should not change drastically within the task area or from the task area to its immediate surroundings. Acceptable uniformity values for task areas are $\geq 0.7$ and for immediate surrounding areas $\geq 0.5$. This metric has an impact on the visual comfort and performance of workers.

$$
U_{o}=\frac{E_{\min }}{E_{\text {average }}}
$$

where:

$E_{\text {min }} \quad$ is the minimum illuminance in the reference area.

$E_{\text {average }}$ is the average illuminance in the reference area.

- Correlated Color Temperature (CTT) and Color Rendering Index $\left(R_{a}\right)$. Correlated color temperature (measured in degrees Kelvin) refers to color appearance of the light emitted by a light source. This affects directly the visual comfort as it affects the way light is perceived by humans and the choice between a cool or warm color temperature should be made based on the direct environment and application. On the other hand, the color rendering index $\left(R_{a}\right)$ indicates the color variation experimented by objects illuminated by a particular light source in comparison to a reference light source. For most visual tasks, light sources with a high color rendering index are preferred (max. value is 100$). R_{a}$ values are usually provided by lamps' manufacturers. 


\section{Visualization and Analysis in Lighting Simulation Software}

This section provides an overview of the techniques used in the lighting design domain to facilitate the analysis of simulation results. We selected two exemplary free lighting simulation software packages and test them against the lighting design for a metal workshop room. We explore and compare the output produced by both packages concerning the way they communicate to the users the results of computing the before mentioned metrics, their ability to validate standards compliance, and the possibility to compare lighting solutions.

\section{Selected Software Packages.}

- DIALux evo 6.2 developed by DIAL GmbH Zentrum für Licht und Smart Building, Germany. This software supports the calculation on of daylight, electric light and energy performance for indoor environments as well as for road projects. The calculation methods used in this package are radiosity (applying an adaptive meshing approach to speed up the computation time) and raytracing (via POV-Ray). The lighting standards for indoor environments supported by DIALux evo are: EN 12464-1, DIN V 18599, and CIE 0972005. More information at: https://www.dial.de/en/dialux/.

- Relux Desktop 2017.1.1.0, developed by Relux Informatik AG, Switzerland. Like DIALux, this software supports the simulation of daylight, electric light and energy performance for electric lighting for three applications: interior and exterior of buildings, road projects, and tunnels. Relux Desktop applies the radiosity and raytracing (based on RADIANCE) calculation methods in its simulation engine. For indoor environments, this software assists the validation of compliance with European lighting standards such as EN 12464-1, EN 1838, ASR A3.4 and DIN 5034. More information at: https://relux.com/en/.

Test Case: Metal Workshop Room. The workshop is divided into five areas or workstations: milling area, lathe station, drilling and grinding area, welding area, and storage area. For simplicity reasons, we assume that the full area of the workshop is destined to rough and average machining (tolerances $\geq 0.1 \mathrm{~mm}$ ). The minimum requirements for this type of visual task as stablished in the European standard EN 12464-1 are described in Table 1.

Table 1. Minimum lighting requirements for Industrial Activities and

Crafts - Metal Working and Processing (EN 12464-1).

\begin{tabular}{|c|c|c|c|}
\hline \multicolumn{4}{|c|}{ Ref no. 5.18.4. Rough and average machining: tolerances $\geq 0.1 \mathrm{~mm}$} \\
\hline $\bar{E}_{m}[l x]$ & $U G R_{L}$ & $U_{o}$ & $\mathrm{R}_{\mathrm{a}}$ \\
\hline 300 & 22 & 0.60 & 60 \\
\hline
\end{tabular}

The lighting design for this room consists of a 3x3 array of architectural LED troffers evenly distributed in the room. The luminaires selected for this test case belong to the CREE Lighting CR Series whose technical specifications are described in Table 2. Since the room does not count with any access to natural light, only electric light is taken into account for the simulation. Figure 1 shows a 3D view, top view, and luminaires arrangement of the designed metal workshop room.
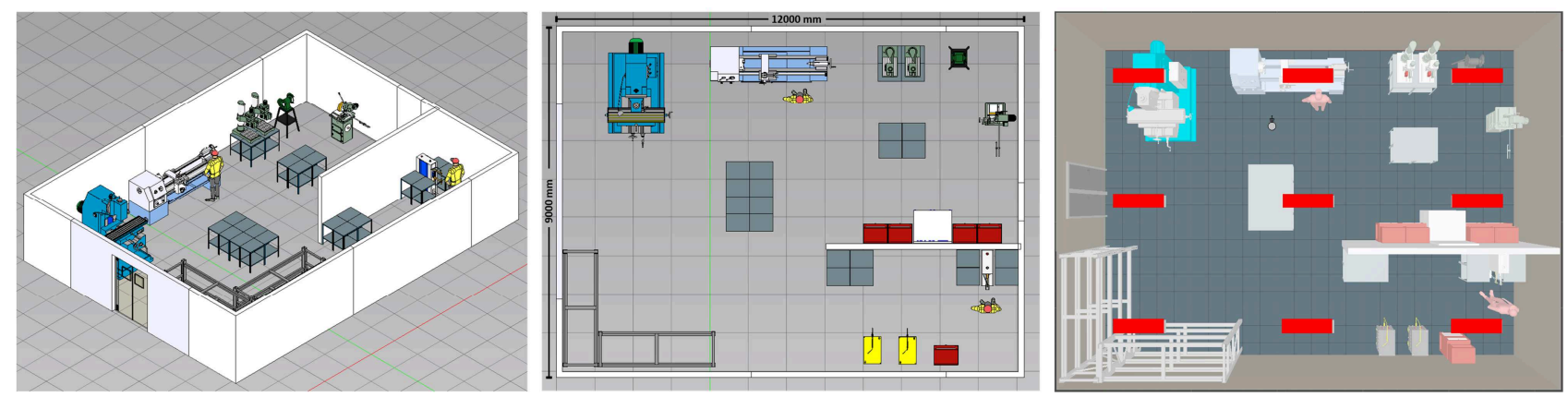

Figure 1. Physical layout of metal workshop room: 3D view (left), top view (middle), and luminaires arrangement (right). 
Table 2. Technical specification for CREE Architectural LED troffers CR14 5000L 30K.

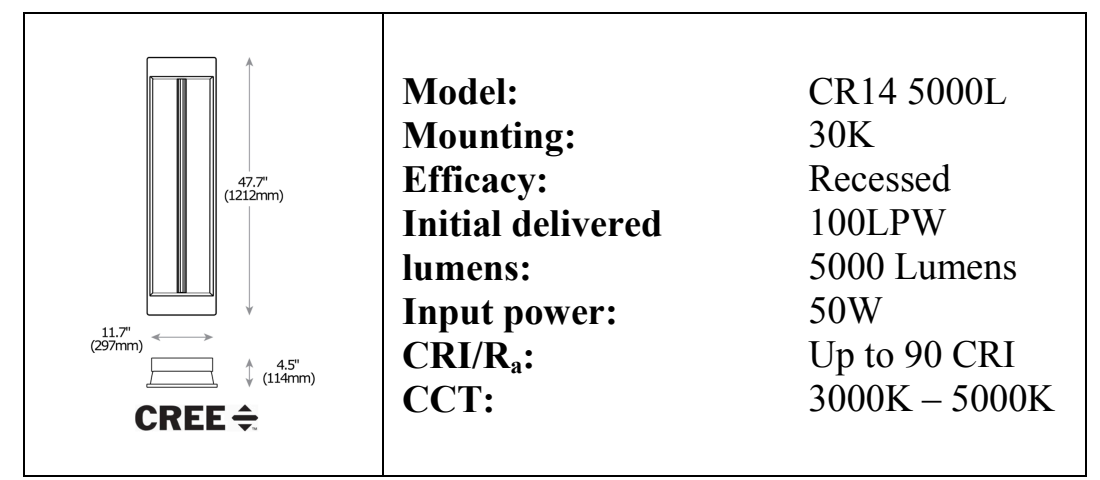

We defined three calculation objects during the simulation setup: 1) a working plane that covers the full area of the room (positioned at 0.8 meters high); 2) a visual tasks area that covers the top surface of the eight tables located at the center of the room (positioned at 0.77 meters high); and 3 ) a calculation point that corresponds to the position of an observer in front of the lathe station for glare calculations (positioned at 1.6 meters high).

Visualization of Simulation Results. In general, most lighting simulation software packages are equipped with a set of basic visualization techniques for the display of simulation results. The difference between packages mainly resides in the possibility to customize this output. Following we present the techniques offered by DIALux evo and ReluxDesktop to visualize the lighting metrics mentioned before. Then, we present in Table 3, a summary of the techniques and their application in both packages. Additionally, we comment on the ability of these tools to compare multiple lighting solutions as well as additional visualizations to facilitate the understanding of particular lighting configurations.

Luminance. DIALux evo and ReluxDesktop offer two alternatives for the visualization of surface luminance: 3D photorealistic renders and false-color maps. While photorealistic renders give a qualitative impression of the overall brightness inside the room, the 3D false-color maps provide a detailed view to the luminance values calculated for different surfaces. Figure 2 shows the visualizations of luminance produced by both packages. 

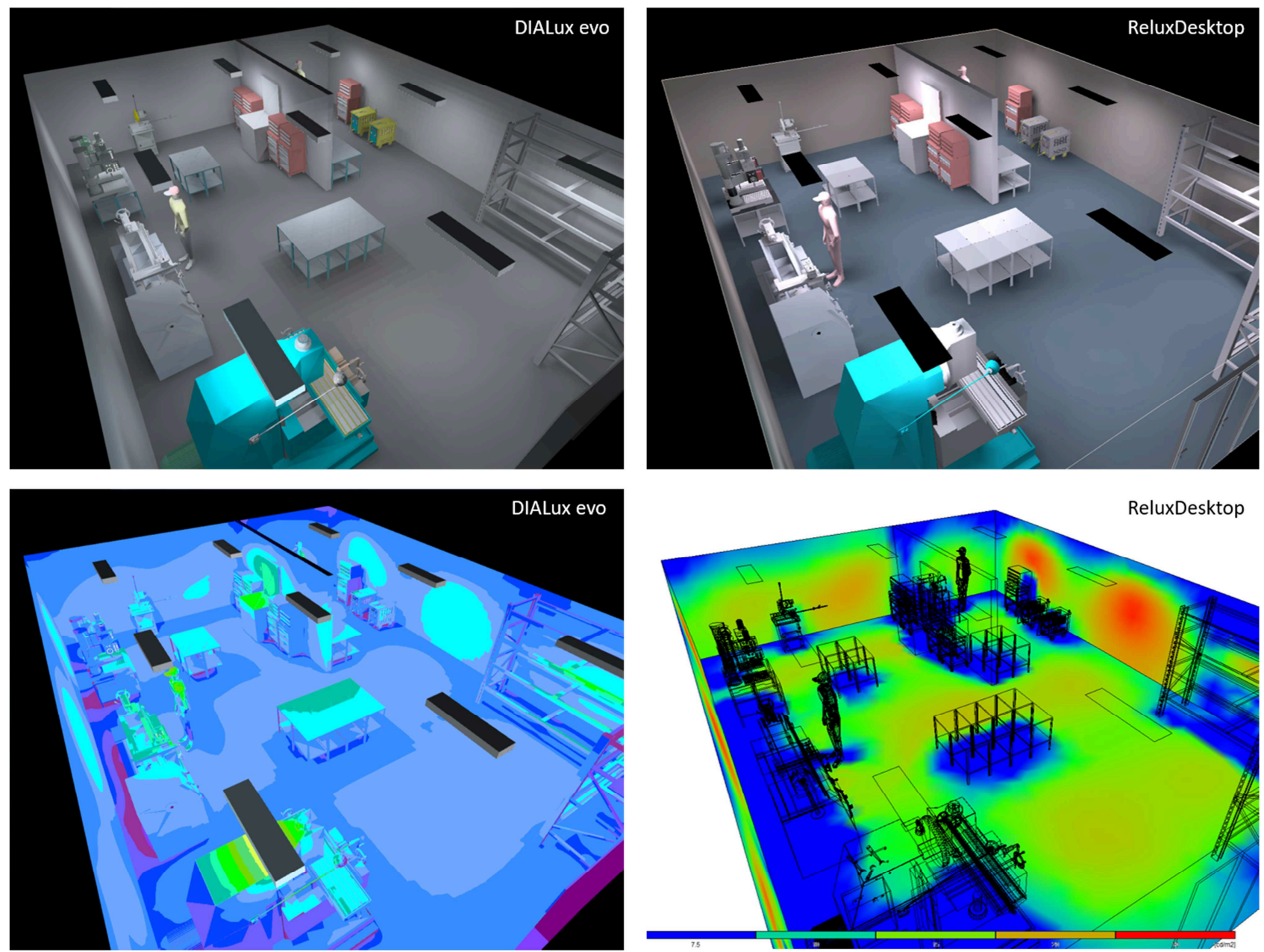

Figure 2. Luminance visualizations in DIALux evo (top-left and bottom-left) and ReluxDesktop (top-right and bottom right).

Illuminance. Three visualization techniques are used in DIALux evo and ReluxDesktop to illustrate the illuminance distribution within calculation objects. Isocontours, which can be described as profiles along which there is a constant illuminance value; false-color maps, which correspond to the mapping of illuminance values to a color function; and value charts displaying illuminance values each point within the calculation objects. Figure 3 illustrates the three techniques applied to visualize the computed illuminance for a calculation object (working plane) positioned at 0.8 meters high. 

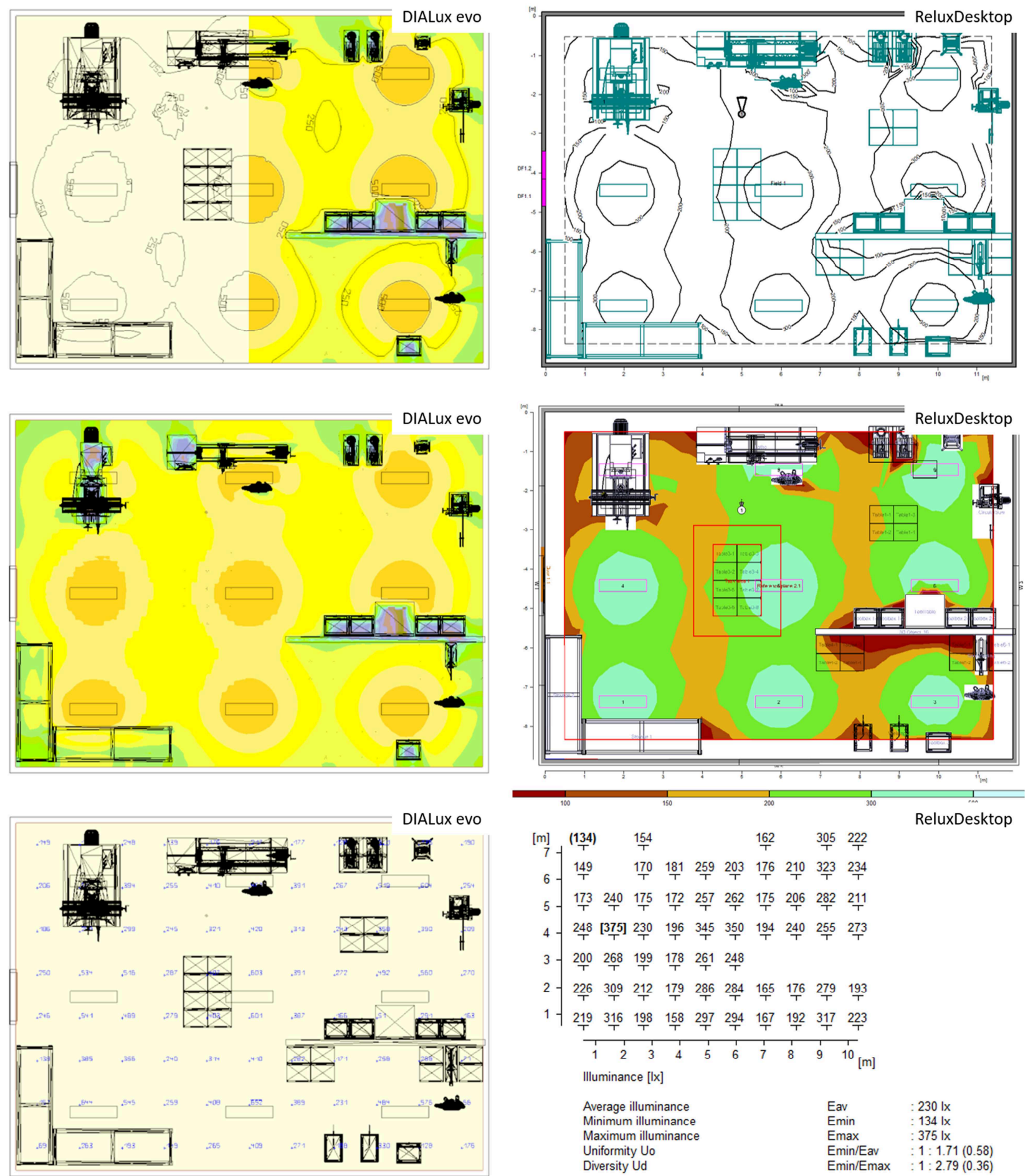

Figure 3. Illuminance distribution visualizations in DIALux evo (left column) and ReluxDesktop (right column) for the working plane at 0.8 meters high. In contrast to ReluxDesktop, DIALux evo allows the combination of visual representations to enhance the readability of the results (top-left).

Unified Glare Rating (UGR). Perceived glare is computed using as reference the position and viewing angle of an observer. The visualization technique used to display the result of this computation in both packages is a radial plot that indicates, for the position and viewing angle being evaluated, the unified glare rating in all directions within the viewing angle range. Figure 4 shows an example of the glare visualization and its complementary views for an observer positioned in front of the lathe area with a viewing angle range of 60 degrees. 

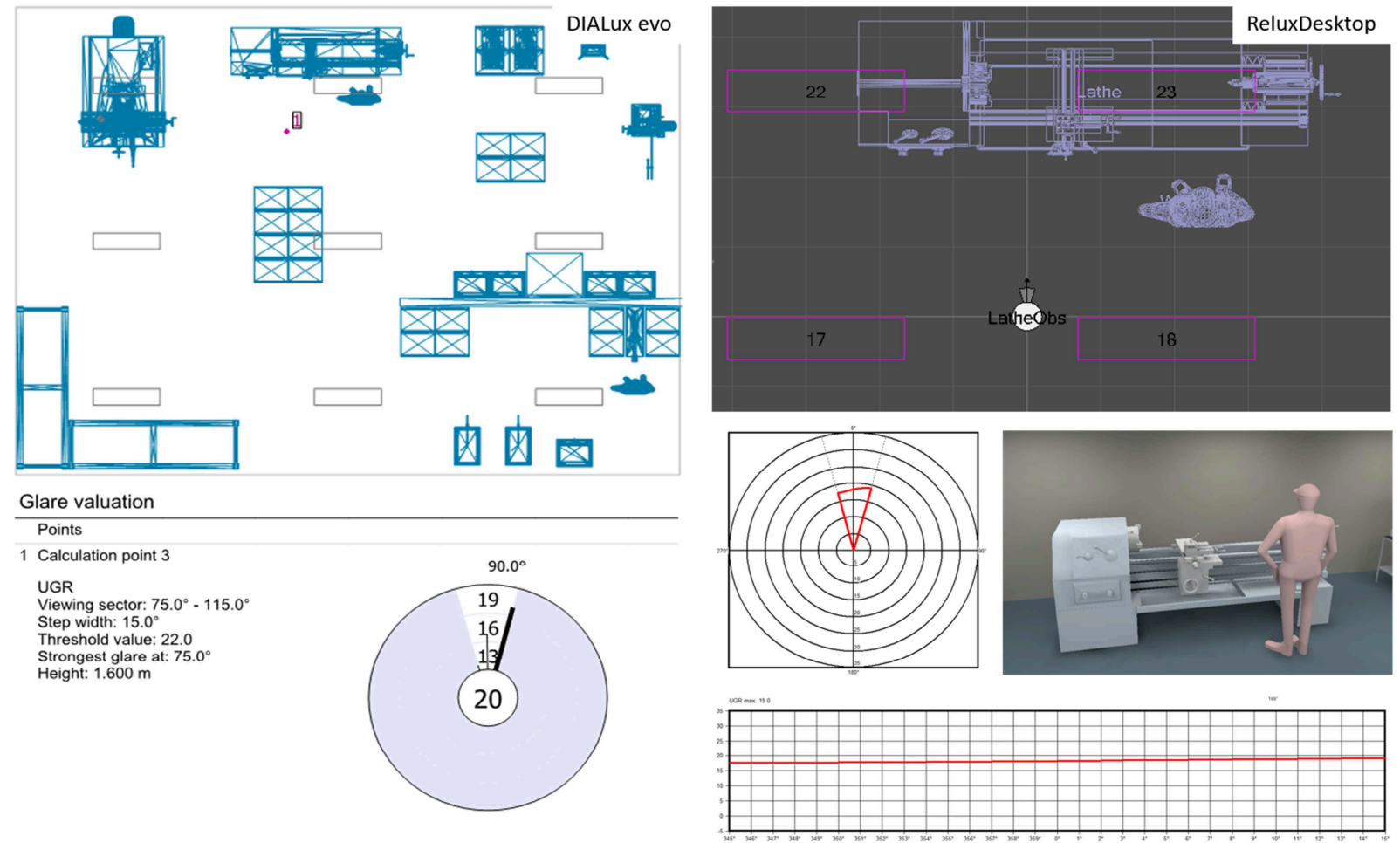

Figure 4. Unified Glare Rating radial plot and complementary views in DIALux evo (left column) and ReluxDesktop (right column). In this case, DIALux evo provides a more intuitive visualization for glare in the evaluated point by indicating at the center of the plot, the maximum glare rating reached at the specified position.

Uniformity Index and Compliance with EN 12464-1. Uniformity and compliance results are presented in a textual report that contains a summary of the most important metrics computed for each calculation object. In addition to the report view, DIALux evo has a results panel in which compliance results for a particular calculation object is indicated with a small square colored in green or red. Figure 5 shows an example of the report and results views for the workplane at 0.8 meter high.
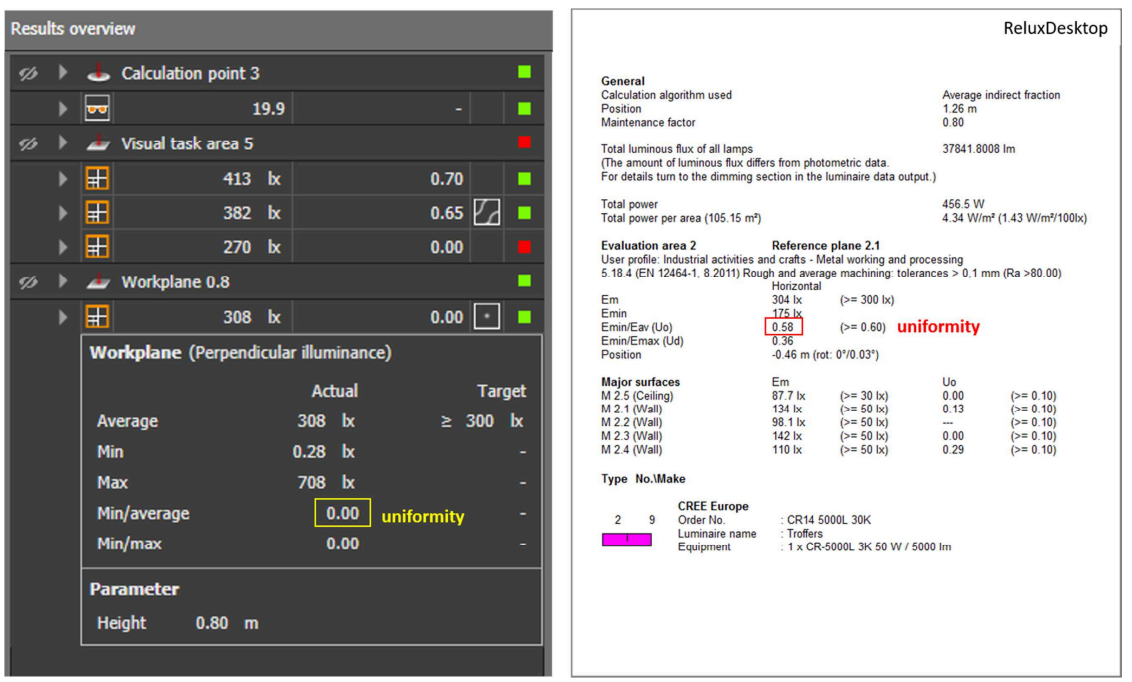

Figure 5. Results panel view in DIALux evo (left) and report view in ReluxDesktop (right). It is worth to mention that the differences in the results are a consequence of the resolution setup for the calculation objects and the way each tool interprets the geometry and materials of the furniture and equipment in the room. We noticed that for the test case, DIALux evo computes illuminance inside the equipment (that is why the minimum illuminance is close to 0 , therefore the global uniformity value is also 0), while ReluxDesktop computes the illuminance around the bounding box of the equipment. 
Correlated Color Temperature and Color Rendering Index. Color temperature can be interactively modified in both packages. To visualize the impact of the color temperature in the room the simulation must be run again. The $3 \mathrm{D}$ rendered view which displays the luminance distribution reveals the effect of changing this parameter. Neither DIALux evo nor ReluxDesktop allow the direct comparison of color temperatures. However, by juxtaposing the images produced in two simulation runs it is possible to see the difference (see Figure 6). As mentioned before, the color rendering index is provided by the manufacturer of the lamp or luminaire. This index is estimated by performing physical measurements under a controlled environment.
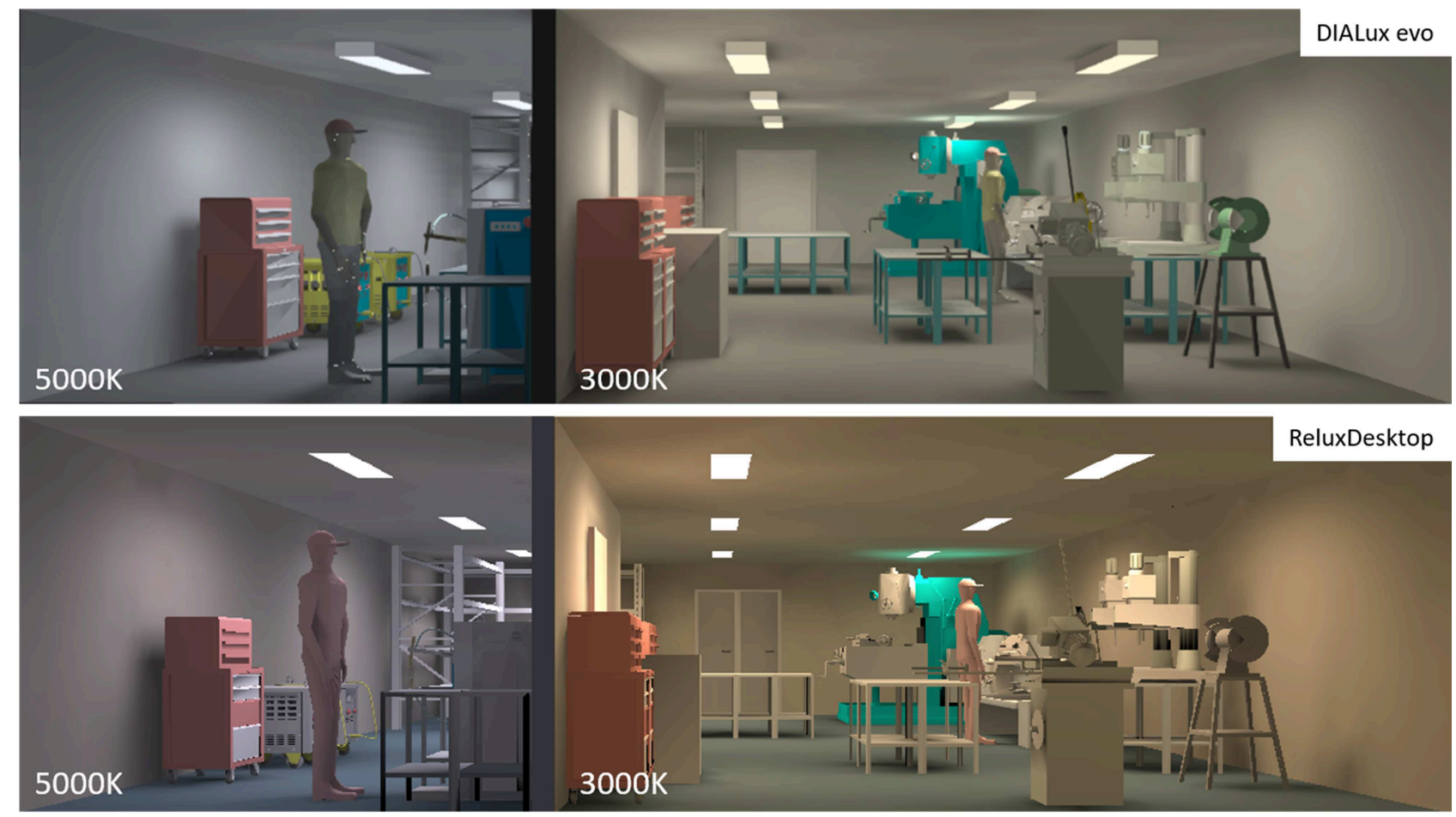

Figure 6. Comparison of color temperatures by juxtaposition of rendered images by DIALux evo (top) and ReluxDesktop (bottom). The left-half and right-half of each picture corresponds to a color temperature of 5000 degrees Kelvin and 3000 degrees Kelvin, respectively.

Table 3. Summary of techniques in DIALux evo / ReluxDesktop for the presentation of simulation results.

\begin{tabular}{|l|l|l|}
\hline Technique & DIALux evo & ReluxDesktop \\
\hline $\begin{array}{l}\text { Photorealistic } \\
\text { renders }\end{array}$ & $\begin{array}{l}\text { Available for the display of luminance } \\
\text { distribution (correlated color } \\
\text { temperature is also observable through } \\
\text { this view) (Figure 2 - top-left and } \\
\text { Figure 6 - top). }\end{array}$ & $\begin{array}{l}\text { Available for the display of luminance } \\
\text { distribution (correlated color } \\
\text { temperature is also observable through } \\
\text { this view) (Figure 2 - top-right and } \\
\text { Figure 6 - bottom). }\end{array}$ \\
\hline Isocontours & $\begin{array}{l}\text { Available for the display of illuminance } \\
\text { distribution on the calculation objects } \\
\text { (e.g., working plane or visual task area) } \\
\text { (Figure 3 - top-left). }\end{array}$ & $\begin{array}{l}\text { Available for the display of luminance } \\
\text { and illuminance distribution on the } \\
\text { calculation objects (e.g., working plane } \\
\text { or visual task area) (Figure 3 - top- } \\
\text { right). }\end{array}$ \\
\hline False-color maps & $\begin{array}{l}\text { Available as 2D maps for the display of } \\
\text { illuminance values on the calculation } \\
\text { objects (Figure 3 - middle-left). } \\
\text { Available as 3D maps depicting the } \\
\text { illuminance distribution on all surfaces } \\
\text { within the room (Figure 2 - bottom- } \\
\text { left). }\end{array}$ & $\begin{array}{l}\text { Available as 2D maps for the display of } \\
\text { illuminance values on the calculation } \\
\text { objects (Figure 3 - middle-right). } \\
\text { Available as 3D maps depicting the } \\
\text { illuminance distribution on the main } \\
\text { surfaces of the room (walls, ceiling, and } \\
\text { floor) (Figure 2 - bottom-right). }\end{array}$ \\
\hline
\end{tabular}




\begin{tabular}{|l|l|l|}
\hline Value charts & $\begin{array}{l}\text { Available for the display of illuminance } \\
\text { values. The spatial context is preserved } \\
\text { via wireframe view of the room (Figure } \\
3 \text { - bottom-left). }\end{array}$ & $\begin{array}{l}\text { Available for the display of luminance } \\
\text { and illuminance values. The spatial } \\
\text { context is provided via plane axes } \\
\text { indicating the dimension of the room } \\
\text { (Figure 3 - bottom-right). }\end{array}$ \\
\hline 2D plots & $\begin{array}{l}\text { Available as radial plot displaying the } \\
\text { UGR for each angle within the viewing } \\
\text { angle range. Accessible only through the } \\
\text { report generation tool (Figure 4 - left). } \\
\text { displaying the UGR for each angle } \\
\text { within the viewing angle range. } \\
\text { Additional information about the } \\
\text { position, orientation, and viewing angle } \\
\text { of the observer can be accessed through } \\
\text { complementary views (Figure 4 - right). }\end{array}$ \\
\hline Summary reports & $\begin{array}{l}\text { Available. Reports are automatically generated including a summary of all } \\
\text { calculated metrics for the whole room and all calculation objects. Reports can be } \\
\text { later exported as a pdf file. Compliance with lighting standards is only indicated in } \\
\text { these reports but it is not integrated into any of the other visualizations (Figure 5). }\end{array}$ \\
\hline Combined views & $\begin{array}{l}\text { Available. Isocontours, false-color maps } \\
\text { and value charts displaying illuminance } \\
\text { distribution can be combined into a } \\
\text { single visualization (Figure 3 - top-left). }\end{array}$ & $\begin{array}{l}\text { Not available. } \\
\text { Visual comparison }\end{array}$ \\
\hline $\begin{array}{l}\text { Not available. The only option to compare lighting alternatives in both tools is } \\
\text { oriented to the energy efficiency of the selected luminaire. It is possible to compare } \\
\text { the costs and energy consumption for multiple luminaires but the option to compare } \\
\text { simulation results for two or more different lighting solutions is not available. }\end{array}$ \\
\hline
\end{tabular}

\section{Discussion and Future Work}

The visualization techniques currently included in most lighting simulation software are ideal to facilitate the global understanding of the lighting conditions for a designed environment. Taking as reference the two lighting simulation software packages tested in this study, we found out that the interactive exploration of the simulation results (filtering, highlighting, aggregation, etc.), integrated visualization of compliance with standards (visualizations within a spatial context), and particularly the direct comparison of design alternatives are aspects open to further improvements. In this section, we discuss how approaches from other domains such as comparative visualization and its applications to image processing and ensemble visualization can be used to address these aspects.

Interactive exploration of simulation results. In information visualization a large amount of tools follow Shneiderman's information seeking mantra: "overview first, zoom and filter, then details on demand" [22] since it suggests and intuitive visual exploration workflow. The support of this workflow, although not the aim of lighting simulation software, can favor the lighting design and decision-making process. Common interaction tasks that might be considered in the implementation of this visual exploration are carefully classified and listed by Yi et al. [23]. Similarly, Kerren and Schreiber [24] offer a compilation of interaction tasks in the framework of visual analytics applications. The authors present an example on the analysis of biological simulations these tasks play an important role supporting the derivation of new knowledge. Specific techniques like multiple coordinated views [25] or dynamic query controls (for example, for interactive specification of features) [26] among others are viable alternatives for supporting interactive exploration of lighting simulation results as evidenced in other applications [27].

Visualization of compliance with standards. As most of the simulation results deriving from lighting simulations are scalar fields, it is easy to interpret them as images in which each pixel stores data on multiple lighting metrics (e.g., luminance, illuminance, uniformity, etc.). Based on this reinterpretation of the results, simple techniques such as image subtraction or weighted 
difference [28] can be used to build quantitative measures for the assessment of global (entire facility) and local (single workstations) quality and compliance with standards. On the other hand, feature extraction and feature-based visualization techniques can be applied for the identification of areas that fulfill or not certain conditions (e.g., identification of extremely dark/bright areas with respect to a standard).

Comparison of lighting solutions. Ensemble visualization techniques can be applied to the analysis of multiple simulation runs with varying input parameters. These techniques range from visual mapping to interactive visual analysis, and computational analysis [29]. Although most of these approaches rely on statistical analysis techniques, the visualization of the results in combination with a context-preserving visualization (namely the visual representation of the space for which a lighting solution is being analyzed) can bring relevant insights about the behavior of a lighting setting under different parameter configurations. Considering that the output of lighting simulations can be treated as image data, mixed approaches integrating image processing and cluster analysis can be applied [30]. Further examples of the application of image processing and computational analysis can be found in [28] [29].

Certainly, the combination of these techniques convey a robust evaluation of multiple lighting solutions against a set of requirements. In addition to this, by enabling not only the global but also local assessment of lighting metrics, experts can tackle directly specific areas of the design which do not comply with the standards. Moreover, the ability to compare multiple lighting solutions towards the identification of a best fit solution for a set of given requirements can also add to experts' knowledge in regards of the behavior of new lighting technologies in different applications. Future work consists of the implementation, integration, and evaluation in practice of these approaches. In addition, we are on the current development of a framework for the task-oriented visual comparison of lighting solutions.

\section{Conclusions}

In this study, we explored the visualization techniques offered by lighting simulation software towards the support of simulation-assisted lighting design processes. We study the visualization and analysis techniques offered by two free lighting simulation software packages: DIALux evo and ReluxDesktop in order to identify the techniques commonly used in the field of lighting design for the visualization and analysis of simulation results. We use a test case of a metal workshop to illustrate how these software packages apply visualization techniques to display different lighting metrics. Based on this exploration, we identified clear opportunities for the improvement of the analysis of these results. We conclude that, although these packages offer a good basis for the analysis of simulation results, three particular aspects require further investigation: 1) interactive exploration of simulation results 2) visualization of global and local compliance with standards, and 3) comparison of design alternatives. Finally, we discuss on how approaches from other domains can help to fill this gap.

\section{Acknowledgements}

This research was funded by the German research foundation (DFG) within the IRTG 2057 "Physical Modeling for Virtual Manufacturing Systems and Processes". 


\section{References}

[1] A. Pellegrino, "Assessment of Artificial Lighting Parameters in a Visual Comfort Perspective," Lighting Research and Technology, vol. 31, no. 3, pp. 107-115, 1999.

[2] C. Chou, C.-C. Lu, and R. Huang, "Effects of different ambient environments on human responses and work performance," Journal of Ambient Intelligence and Humanized Computing, vol. 7, no. 6, pp. 1-10, 2016.

[3] K. Papamichael, M. Siminovitch, J. A. Veitch, and L. Whitehead, "High Color Rendering Can Enable Better Vision without Requiring More Power," LEUKOS - Journal of Illuminating Engineering Society of North America, vol. 12, no. 1-2, pp. 27-38, 2016.

[4] Comité Européen De Normalisation, "Light and Lighting - Lighting of Workplaces - Part 1: Indoor Work Places. EN 12464-1," 2011.

[5] Standards Australia, "Interior and Workplace Lighting - Part 1: General Principles and Recommendations. AS/NZS 1680.1:2006," 2006.

[6] International Organization for Standardization, "Lighting of work places — Part 1: Indoor. ISO 8995-1:2002," 2002.

[7] T. Hong, S. . Chou, and T. . Bong, "Building simulation: an overview of developments and information sources," Building and Environment, vol. 35, no. 4, pp. 347-361, May 2000.

[8] D. E. Christakou and C. N. D. Amorim, "Daylight Simulation: Comparison of Softwares for Architect's Utilization," in IBPSA Proceedings: Building Simulation Conference, 2005, pp. 183-190.

[9] C. F. Reinhart and S. Herkel, "The Simulation of Annual Daylight Illuminance DistributionsA State-of-the-Art Comparison of Six RADIANCE-based Methods," Energy and Buildings, vol. 32, no. 2, pp. 167-187, 2000.

[10] S. Attia, L. Beltran, A. de Herde, and J. L. M. Hensen, "'Architect Friendly': a comparison of ten different building performance simulation tools," in IBPSA Proceedings: Building Simulation Conference, 2009, pp. 204-211.

[11] G. G. Roy, "A Comparative Study of Lighting Simulation Packages Suitable for use in Architectural Design,” 2000.

[12] J. Ashmore and P. Richens, "Computer Simulation in Daylight Design: a comparison.," Architectural Science Review, vol. 44, no. 1, pp. 33-44, 2001.

[13] C. E. Ochoa, M. B. C. Aries, and J. L. M. Hensen, "State of the art in lighting simulation for building science: a literature review," Journal of Building Performance Simulation, vol. 5, no. 4, pp. 209-233, 2012.

[14] A. Iversen, N. Roy, M. Hvass, M. Jørgensen, J. Christoffersen, W. Osterhaus, and K. Johnsen, Daylight Calculations in Practice: An Investigation of the Ability of Nine Daylight Simulation Programs to Calculate the Daylight Factor in Five Typical Rooms, 1st Editio. SBI, 2013.

[15] S. H. Shikder, A. D. F. Price, and M. Mourshed, "Evaluation of four artificial lighting simulation tools with virtual building reference," in Proceedings of the European Simulation and Modelling Conference (ESM), 2009, pp. 77-82.

[16] M. S. Ubbelohde and C. Humann, "Comparative Evaluation of Four Daylighting Software Programs," ACEE Summer Study on Energy Efficiency in Buildings, vol. 1988, p. 16, 1998.

[17] P. L. and C. J. A., "Communicating Building Simulation Outputs to Users," in Proceedings of the Eighth International IBPSA Conference, August 11-14, 2005, Eindhoven, Netherlands, 2003. 
[18] S. Srivastav, S. Lannon, D. K. Alexander, and P. Jones, "A Review and Comparison of Data Visualization Techniques Used in Building Design and in Building Simulation," in IBPSA Proceedings: Building Simulation Conference, 2009, pp. 1942-1949.

[19] K. Haeb, S. Schweitzer, D. Fernandez Prieto, E. Hagen, D. Engel, M. Bottinger, and I. Scheler, "Visualization of Building Performance Simulation Results: State-of-the-Art and Future Directions," 2014 IEEE Pacific Visualization Symposium, pp. 311-315, Mar. 2014.

[20] National Framework for Energy Efficiency - Australia, "Training Guide: The Basics of Efficient Lighting." 2009.

[21] Zumtobel Lighting GmbH, The Lighting Handbook, 4th Edition. Dornbirn, Austria, 2013.

[22] B. Shneiderman, "The Eyes Have It: A Task by Data Type Taxonomy for Information Visualizations," in Proceedings 1996 IEEE Symposium on Visual Languages, 1996, pp. 336343.

[23] J. Yi, Y. A. Kang, and J. Stasko, "Toward a Deeper Understanding of the Role of Interaction in Information Visualization," IEEE Transactions on Visualization and Computer Graphics, vol. 13, no. 6, pp. 1224-31, 2007.

[24] A. Kerren and F. Schreiber, "Toward the Role of Interaction in Visual Analytics," in Proceedings of the Winter Simulation Conference, 2012, p. 420.

[25] J. C. Roberts, "State of the Art: Coordinated Multiple Views in Exploratory Visualization," in CMV '07 Proceedings of the Fifth International Conference on Coordinated and Multiple Views in Exploratory Visualization, 2007, pp. 61-71.

[26] C. Ahlberg, C. Williamson, and B. Shneiderman, "Dynamic Queries for Information Exploration: An Implementation and Evaluation," in Proceedings of the SIGCHI conference on Human factors in computing systems - CHI '92, 1992, pp. 619-626.

[27] H. Doleisch, M. Gasser, and H. Hauser, "Interactive feature specification for focus + context visualization of complex simulation data," VISSYM '03 Proceedings of the Symposium on Data Visualisation, pp. 239-249, 2003.

[28] R. C. Gonzalez and R. E. Woods, Digital Image Processing, 3rd Editio. Pearson Prentice Hall, 2008.

[29] J. Kehrer and H. Hauser, "Visualization and Visual Analysis of Multifaceted Scientific Data: A Survey," IEEE Transactions on Visualization and Computer Graphics, vol. 19, no. 3, pp. 495-513, 2013.

[30] J. Schmidt, M. E. Groller, and S. Bruckner, "VAICo: Visual analysis for image comparison," IEEE Transactions on Visualization and Computer Graphics, vol. 19, no. 12, pp. 2090-2099, 2013.

[31] H.-G. Pagendarm and F. H. Post, "Comparative Visualization: Approaches and Examples," Visualization in Scientific Computing, pp. 95-108, 1995.

[32] T. Nocke, M. Flechsig, and U. Bohm, "Visual exploration and evaluation of climate-related simulation data," in Simulation Conference, 2007 Winter, 2007, pp. 703-711. 